\title{
EQUIVARIANT DIXMIER-DOUADY CLASSES
}

\author{
Mathieu StiÉnON
}

\begin{abstract}
An equivariant bundle gerbe à la Meinrenken over a $G$-manifold $M$ is known to be a special type of $S^{1}$-gerbe over the differentiable stack $[M / G]$. We prove that the natural morphism relating the Cartan and simplicial models of equivariant cohomology in degree 3 maps the Dixmier-Douady class of an equivariant bundle gerbe à la Meinrenken to the Behrend-Xu-Dixmier-Douady class of the corresponding $S^{1}$-gerbe.
\end{abstract}

\section{Introduction}

Recently, following Brylinski's pioneering work [2, there has been increasing interest in studying the differential geometry of gerbes. In particular, Murray defined and investigated bundle gerbes [13, which were further studied by Chatterjee [4] and Hitchin 9 .

By definition, a bundle gerbe over a smooth manifold $M$ is a central $S^{1}$-extension of the groupoid $X \times_{M} X \rightrightarrows X$ coming from a surjective submersion $X \stackrel{\pi}{\rightarrow} M$. A class in $H^{3}(M, \mathbb{Z})$ is associated to any bundle gerbe over $M$. It is called the Dixmier-Douady (DD) class. The bundle gerbes over $M$ are classified, up to Morita equivalence (a.k.a. stable equivalence [14]), by their DD classes.

Moreover, like the Chern classes of $S^{1}$-bundles, the DD classes can be expressed, up to torsion elements, in terms of the 3 -curvature. The equivariant counterparts of bundle gerbes are called equivariant bundle gerbes [11. They are $G$-equivariant central $S^{1}$-extensions of a groupoid $X \times_{M} X \rightrightarrows X$ associated to a $G$-equivariant surjective submersion $X \stackrel{\pi}{\rightarrow} M$. Meinrenken [12] and Gawędzki-Reis [7] studied extensively the equivariant bundle gerbes over simple Lie groups. In [12, Meinrenken introduced the equivariant 3-curvature of an equivariant bundle gerbe. It is a closed equivariant 3-form in Cartan's model of equivariant cohomology. Its cohomology class corresponds to the equivariant DD class of the gerbe.

Recently, Behrend-Xu studied $S^{1}$-gerbes over differentiable stacks. From their perspective, a $G$-equivariant bundle gerbe is an $S^{1}$-gerbe over the quotient stack $[M / G]$, which is a Morita equivalence class of central $S^{1}$-extensions of groupoids $\tilde{H} \rightarrow H \rightrightarrows N$, where $H \rightrightarrows N$ is Morita equivalent to the groupoid $M \rtimes G \rightrightarrows M$. From connection type data on such a central $S^{1}$-extension, Behrend-Xu construct a characteristic class in the degree 3 cohomology de Rham cohomology group $H_{\mathrm{DR}}^{3}\left(H_{\text {• }}\right)$ of the corresponding simplicial manifold.

The purpose of this paper is to establish an explicit connection between equivariant bundle gerbes à la Meinrenken and $S^{1}$-gerbes over $[M / G]$ à la Behrend-Xu. For this purpose, we use an explicit map, obtained by Bursztyn-Crainic-Weinstein-Zhu [3], between the Cartan and simplicial models of equivariant cohomology in degree 3. As

Received by the editors May 1, 2008. 
a byproduct, we establish some further properties of the BCWZ-map and show that it is indeed an isomorphism at the cohomology level. We hope that this result will be of independent interest.

Our main theorem states that the BCWZ isomorphism maps the Meinrenken equivariant DD class of a $G$-equivariant bundle gerbe over a $G$-manifold $M$ to the Behrend$\mathrm{Xu}$-Dixmier-Douady (BXDD) class of the corresponding $S^{1}$-gerbe over the quotient stack $[M / G]$.

The paper is organized as follows.

Section 3.1 recalls the definition of equivariant bundle gerbes and equivariant central $S^{1}$-extensions, while Section 3.2 recalls how the Dimier-Douady (DD) class of a $G$-equivariant bundle gerbe may be computed from connection type data.

Section 4.1 gives a brief account of $S^{1}$-gerbes $\tilde{\mathfrak{X}}$ over a differentiable stack $\mathfrak{X}$ and their DD classes. The DD class of an $S^{1}$-gerbe induces a degree 3 de Rham cohomology class called BXDD class which can be computed from connection type data.

In Section 4.2, we explain how an equivariant bundle gerbe over a $G$-manifold $M$ (in the sense of Murray and Meinrenken) produces an $S^{1}$-gerbe over the stack $[M / G]$ (in the sense of Behrend-Xu). And we compute the BXDD class of the central $S^{1}$-extension of groupoids presenting the $S^{1}$-gerbe over $[M / G]$ associated to a $G$ equivariant bundle gerbe over the manifold $M$.

In Section 5.1, we discuss the explicit formula due to BCWZ relating the Cartan and simplicial models of equivariant cohomology in degree 3 and detail some additional properties of this map.

Sections 5.2 and 5.3 contain our main result.

\section{Preliminaries}

We start by recalling a few definitions and conventions used throughout this paper.

Let $G$ be a compact connected Lie group. A $G$-manifold is a smooth manifold $M$ endowed with a right action of $G$, which is denoted by the symbol $\star, M \times G:(m, g) \mapsto$ $m \star g$. If $M$ is a $G$-manifold and $\xi \in \mathfrak{g}=\operatorname{Lie}(G)$, then $\widehat{\xi}$ denotes the infinitesimal vector field on $M$ defined by the relation $\left.\widehat{\xi}\right|_{x}=\left.\frac{d}{d t} x \star e^{t \xi}\right|_{0}, \quad \forall x \in M$.

The Cartan model for equivariant cohomology is the differential complex $\left(\Omega_{G}^{\bullet}(M), d_{G}\right)$ defined by

$$
\Omega_{G}^{k}(M)=\bigoplus_{2 i+j=k}\left(S^{i} \mathfrak{g}^{*} \otimes \Omega^{j}(M)\right)^{G}
$$

and

$$
\left.\left(d_{G} \alpha\right)(\xi)=d(\alpha(\xi))-\widehat{\xi}\right\lrcorner \alpha(\xi),
$$

where $\xi \in \mathfrak{g}$ and the element $\alpha$ of $\Omega_{G}^{\bullet}(M)$ is seen as an $\Omega^{\bullet}(M)$-valued polynomial on $\mathfrak{g}$.

The multiplication of a Lie groupoid $\Gamma_{1} \rightrightarrows \Gamma_{0}$ is denoted by $\Gamma_{2} \rightarrow \Gamma_{1}:(x, y) \mapsto x \cdot y$, where $\Gamma_{2}:=\left\{(x, y) \in \Gamma_{1} \times \Gamma_{1} \mid t(x)=s(y)\right\}$.

By a $G$-groupoid, we mean a Lie groupoid $\Gamma_{1} \rightrightarrows \Gamma_{0}$ such that both $\Gamma_{1}$ and $\Gamma_{0}$ are $G$-manifolds and all the structure maps $(s, t, m, \iota, \varepsilon)$ are $G$-equivariant. Recall that any Lie groupoid $\Gamma_{1} \rightrightarrows \Gamma_{0}$ gives rise to a simplicial manifold

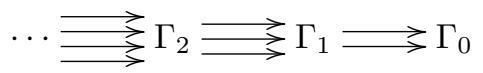


where

$$
\Gamma_{n}=\left\{\left(x_{1}, \ldots, x_{n}\right) \mid t\left(x_{i}\right)=s\left(x_{i+1}\right), i=1, \ldots, n-1\right\}
$$

is the set of composable $n$-tuples of elements of $\Gamma_{1}$ and the face maps $\varepsilon_{i}^{n}: \Gamma_{n} \rightarrow \Gamma_{n-1}$ are given, for $n>1$, by

$$
\begin{aligned}
& \varepsilon_{0}^{n}\left(x_{1}, x_{2}, \ldots, x_{n}\right)=\left(x_{2}, \ldots, x_{n}\right) \\
& \varepsilon_{n}^{n}\left(x_{1}, x_{2}, \ldots, x_{n}\right)=\left(x_{1}, \ldots, x_{n-1}\right) \\
& \varepsilon_{i}^{n}\left(x_{1}, x_{2}, \ldots, x_{n}\right)=\left(x_{1}, \ldots, x_{i} x_{i+1}, \ldots, x_{n}\right), \quad 1 \leq i \leq n-1,
\end{aligned}
$$

and, for $n=1$, by $\varepsilon_{0}^{1}(x)=t(x)$ and $\varepsilon_{1}^{1}(x)=s(x)$. They satisfy the simplicial relations

$$
\varepsilon_{i}^{n-1} \circ \varepsilon_{j}^{n}=\varepsilon_{j-1}^{n-1} \circ \varepsilon_{i}^{n} \quad \forall i<j .
$$

See [15, 6] for more details.

Given a Lie groupoid $\Gamma_{1} \rightrightarrows \Gamma_{0}$, consider the double complex $\Omega^{\bullet}\left(\Gamma_{\bullet}\right)$ :

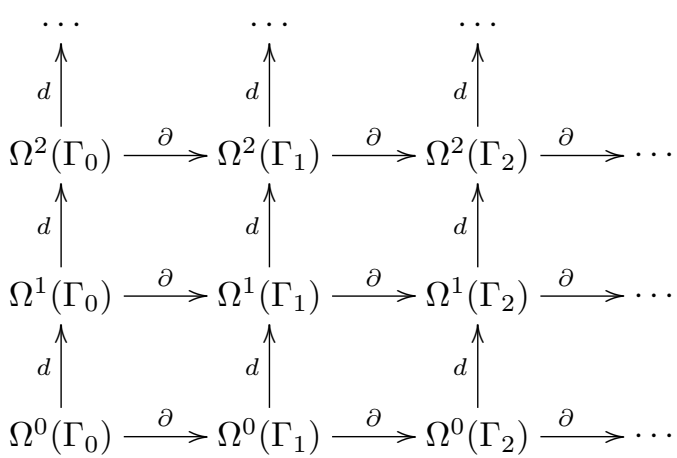

Its coboundary maps are $d: \Omega^{k}\left(\Gamma_{p}\right) \rightarrow \Omega^{k+1}\left(\Gamma_{p}\right)$, the usual exterior differential of smooth forms, and $\partial: \Omega^{k}\left(\Gamma_{p}\right) \rightarrow \Omega^{k}\left(\Gamma_{p+1}\right)$, the alternating sum of the pullbacks by the face maps:

$$
\partial=\sum_{i=0}^{n}(-1)^{i}\left(\varepsilon_{i}^{n}\right)^{*}
$$

We denote the total differential by $D=(-1)^{p} d+\partial$. The cohomology groups

$$
H_{\mathrm{DR}}^{k}\left(\Gamma_{\bullet}\right):=H^{k}\left(\Omega^{\bullet}\left(\Gamma_{\bullet}\right), D\right)
$$

of the total complex $\left(\Omega_{\mathrm{DR}}^{\bullet}\left(\Gamma_{\bullet}\right), D\right)$ (where $\left.\Omega_{\mathrm{DR}}^{k}\left(\Gamma_{\bullet}\right)=\bigoplus_{i=0}^{k} \Omega^{k-i}\left(\Gamma_{i}\right)\right)$ are called the de Rham cohomology groups of the groupoid $\Gamma_{1} \rightrightarrows \Gamma_{0}$. 
Now let $\Gamma_{1} \rightrightarrows \Gamma_{0}$ be a $G$-groupoid. We can consider the double complex $\Omega_{G}^{\bullet}\left(\Gamma_{\bullet}\right)$ :

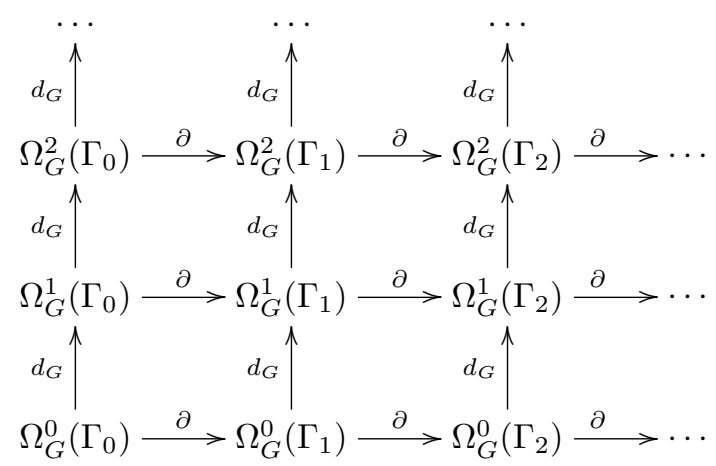

Its coboundary operators are $d_{G}: \Omega_{G}^{k}\left(\Gamma_{p}\right) \rightarrow \Omega_{G}^{k+1}\left(\Gamma_{p}\right)$, the differential operator of the Cartan model, and $\partial: \Omega_{G}^{k}\left(\Gamma_{p}\right) \rightarrow \Omega_{G}^{k}\left(\Gamma_{p+1}\right)$, the natural extension of (1). We denote the total differential by $D_{G}=(-1)^{p} d_{G}+\partial$. The cohomology groups

$$
H_{G}^{k}\left(\Gamma_{\bullet}\right):=H^{k}\left(\Omega_{G}^{\bullet}\left(\Gamma_{\bullet}\right), D_{G}\right)
$$

of the total complex are called the equivariant cohomology groups of the G-groupoid $\Gamma_{1} \rightrightarrows \Gamma_{0}$. See [10].

\section{Equivariant bundle gerbes à la Meinrenken}

In this section, we recall the notion of equivariant bundle gerbes and their equivariant Dixmier-Douady classes in terms of the Cartan model. We closely follow Meinrenken's approach [12].

3.1. Equivariant central $S^{1}$-extensions. Assume that $X \stackrel{\pi}{\rightarrow} M$ is a surjective submersion. Consider the Lie groupoid

$$
\Gamma \rightrightarrows X, \quad \text { with } \Gamma=X \times_{M} X,
$$

the source and target maps $s(x, y)=x$ and $t(x, y)=y$, and the multiplication

$$
(x, y) \cdot(y, z)=(x, z) .
$$

Then we have the Morita morphism [1]

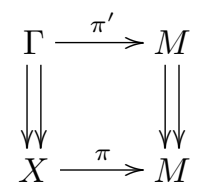

where $\pi^{\prime}: \Gamma \rightarrow M$ is the map $(x, y) \mapsto \pi(x)=\pi(y)$. Indeed $\Gamma \rightrightarrows X$ is the pullback of the trivial groupoid $M \rightrightarrows M$ to $X$ through $\pi$.

Furthermore, if $G$ is a Lie group, $X$ and $M$ are $G$-manifolds and $X \stackrel{\pi}{\rightarrow} M$ is a $G$-equivariant surjective submersion, it is clear that the Lie group $G$ acts on $\Gamma \rightrightarrows X$ by groupoid automorphisms (i.e. $\Gamma \rightrightarrows X$ is a $G$-groupoid) and that $\pi^{\prime}$ in $(3)$ is a $G$-equivariant Morita morphism. 
Recall that a central $S^{1}$-extension of a Lie groupoid $H \rightrightarrows N$ consists of a morphism of Lie groupoids

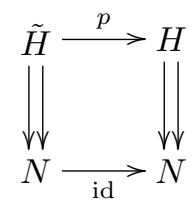

and a left $S^{1}$-action on $\tilde{H}$, making $p: \tilde{H} \rightarrow H$ a (left) principal $S^{1}$-bundle [17, 1]. These two structures are compatible in the following sense:

$$
\left(\lambda_{1} \tilde{x}\right) \cdot\left(\lambda_{2} \tilde{y}\right)=\lambda_{1} \lambda_{2}(\tilde{x} \cdot \tilde{y}),
$$

for all $\lambda_{1}, \lambda_{2} \in S^{1}$ and $(\tilde{x}, \tilde{y}) \in \tilde{H}_{2}:=\tilde{H} \times_{t, N, s} \tilde{H}$.

We will use the shorthand notation $\tilde{H} \stackrel{p}{\rightarrow} H \rightrightarrows N$ to denote the above central $S^{1}$-extension.

A central $S^{1}$-extension $\tilde{H} \stackrel{p}{\rightarrow} H \rightrightarrows N$ is said to be $G$-equivariant if both $\tilde{H} \rightrightarrows N$ and $H \rightrightarrows N$ are $G$-groupoids, the groupoid morphism $p: \tilde{H} \rightarrow H$ in (4) is $G$ equivariant and the $G$-action preserves the principal $S^{1}$-bundle $\tilde{H} \stackrel{p}{\rightarrow} H$. That is, if the following relations:

$$
\begin{gathered}
(\tilde{x} \cdot \tilde{y}) \star g=(\tilde{x} \star g) \cdot(\tilde{y} \star g) \\
p(\tilde{x} \star g)=p(\tilde{x}) \star g \\
(\lambda \tilde{x}) \star g=\lambda(\tilde{x} \star g)
\end{gathered}
$$

are satisfied for all $g \in G$, all composable pairs $(\tilde{x}, \tilde{y})$ in $\tilde{H}_{2}$ and all $\lambda \in S^{1}$.

Bundle gerbes were invented by Murray [13] (see also [9, 4]). By definition, a bundle gerbe over a manifold $M$ is a central $S^{1}$-extension of the Lie groupoid $\Gamma \rightrightarrows X$ (as in (2p) obtained from a surjective submersion $X \rightarrow M$. There is a natural equivalence relation on central $S^{1}$-extensions [1, 17, the so-called Morita equivalence (or stable equivalence in [14]), whose equivalence classes are classified by the cohomology group $H^{3}(M, \mathbb{Z})$. The class in $H^{3}(M, \mathbb{Z})$ attached to a central $S^{1}$-extension is called its Dixmier-Douady class. Equivariant bundle gerbes are equivariant counterparts of bundle gerbes. According to Meinrenken 12, a $G$-equivariant bundle gerbe over a $G$-manifold $M$ is a $G$-equivariant central $S^{1}$-extension of the groupoid $\Gamma \rightrightarrows X$ associated to a $G$-equivariant surjective submersion $X \rightarrow M$ as in (2).

3.2. Equivariant Dixmier-Douady classes. Below we recall Meinrenken's definition of the equivariant 3-curvature and equivariant Dixmier-Douady class of a $G$ equivariant bundle gerbe 1

Definition 3.1. Let $\widetilde{\Gamma} \stackrel{p}{\rightarrow} \Gamma \rightrightarrows X$ be a G-equivariant bundle gerbe, where $X \stackrel{\pi}{\rightarrow} M$ is a $G$-equivariant surjective submersion and $\Gamma=X \times_{M} X$ is the resulting groupoid as in 2 .

\footnotetext{
${ }^{1}$ What Meinrenken called an "equivariant connection" 12 consists of both an equivariant connection and an equivariant curving in our terminology.
} 
(a) An equivariant connection is a G-invariant 1-form $\theta \in \Omega^{1}(\widetilde{\Gamma})^{G}$ such that $\theta$ is a connection 1 -form for the principal $S^{1}$-bundle $\widetilde{\Gamma} \stackrel{p}{\rightarrow} \Gamma$ and satisfies

$$
\widetilde{\partial} \theta=0 .
$$

(b) Given an equivariant connection $\theta$, an equivariant curving is a degree 2 element $B_{G} \in \Omega_{G}^{2}(X)$ such that

$$
\operatorname{curv}_{G}(\theta)=\partial B_{G},
$$

where $\operatorname{curv}_{G}(\theta)$ denotes the equivariant curvature of the $S^{1}$-principal bundle $\widetilde{\Gamma} \stackrel{p}{\rightarrow} \Gamma$, i.e. the element $\operatorname{curv}_{G}(\theta) \in \Omega_{G}^{2}(\Gamma)$ characterized by the relation

$$
d_{G} \theta=p^{*} \operatorname{curv}_{G}(\theta) .
$$

(c) Given an equivariant connection and an equivariant curving $\left(\theta, B_{G}\right)$, the corresponding equivariant 3-curvature is the equivariant 3-form $\eta_{G} \in \Omega_{G}^{3}(M)$ such that

$$
\pi^{*} \eta_{G}=d_{G} B_{G}
$$

Here the coboundary operators associated to the groupoids $\Gamma \rightrightarrows X$ and $\widetilde{\Gamma} \rightrightarrows X$ as in (1) are denoted $\partial$ and $\widetilde{\partial}$ respectively.

The following result seems to be standard (see [12, 16]). However, we could not find a complete proof in the literature. For the sake of completeness, we will sketch a proof below.

Proposition 3.2. Let $\widetilde{\Gamma} \stackrel{p}{\rightarrow} \Gamma \rightrightarrows X$ be a G-equivariant bundle gerbe over a $G$-manifold $M$.

(a) Equivariant connections and curvings $\left(\theta, B_{G}\right)$ always exist.

(b) The class $\left[\eta_{G}\right] \in H_{G}^{3}(M)$ defined by the equivariant 3-curvature is independent of the choice of $\theta$ and $B_{G}$.

We need the following lemma.

Lemma 3.3. (a) Given a surjective submersion $\pi: X \rightarrow M$, the sequence

$$
0 \rightarrow \Omega^{k}(M) \stackrel{\pi^{*}}{\longrightarrow} \Omega^{k}(X) \stackrel{\partial}{\rightarrow} \Omega^{k}(\Gamma) \stackrel{\partial}{\rightarrow} \Omega^{k}\left(\Gamma_{2}\right) \stackrel{\partial}{\rightarrow} \cdots
$$

is exact.

(b) Given two $G$-manifolds $X$ and $M$ and a $G$-equivariant surjective submersion $\pi: X \rightarrow M$, the sequence

$$
0 \rightarrow \Omega^{k}(M)^{G} \stackrel{\pi^{*}}{\longrightarrow} \Omega^{k}(X)^{G} \stackrel{\partial}{\rightarrow} \Omega^{k}(\Gamma)^{G} \stackrel{\partial}{\rightarrow} \Omega^{k}\left(\Gamma_{2}\right)^{G} \stackrel{\partial}{\rightarrow} \cdots
$$

is exact.

(c) Given a G-equivariant surjective submersion $\pi: X \rightarrow M$ between two $G$ manifolds $X$ and $M$, the sequence

$$
0 \rightarrow \Omega_{G}^{k}(M) \stackrel{\pi^{*}}{\longrightarrow} \Omega_{G}^{k}(X) \stackrel{\partial}{\rightarrow} \Omega_{G}^{k}(\Gamma) \stackrel{\partial}{\rightarrow} \Omega_{G}^{k}\left(\Gamma_{2}\right) \stackrel{\partial}{\rightarrow} \cdots
$$

is exact. 
Proof. (a) This was proved in [13].

(b) As is customary, we use the symbol $R_{g}$ to denote the action (from the right) of an element $g \in G$ on a $G$-manifold. Since the face maps of the simplicial manifold

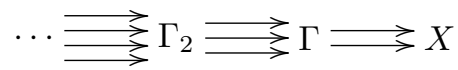

(and $X \stackrel{\pi}{\rightarrow} M$ ) are all $G$-equivariant, $R_{g}^{*}$ commutes with $\partial$ (and $\pi^{*}$ ). Hence 9 is a subcomplex of (8). Now $\pi^{*}$ in $(9)$ is a restriction of $\pi^{*}$ in (8), which is injective. Therefore, $\pi^{*}$ in $(9)$ is injective. Finally, take $\omega \in \Omega^{k}\left(\Gamma_{p}\right)^{G} \subset \Omega^{k}\left(\Gamma_{p}\right)$ such that $\partial \omega=0$. By (a), there exists $\nu \in \Omega^{k}\left(\Gamma_{p-1}\right)$ such that $\partial \nu=\omega$. Since the group $G$ is compact, we can choose a left-invariant Haar measure $d g$ on $G$ and define a $G$-invariant k-form $\nu^{\prime}$ which satisfies $\partial \nu^{\prime}=\omega$ by

$$
\nu^{\prime}=\frac{1}{V} \int_{G} R_{g}^{*} \nu d g \in \Omega^{k}\left(\Gamma_{p-1}\right)^{G},
$$

where $V=\int_{G} 1 d g$ is the total volume of $G$.

(c) For simplicity, we only consider the case $k=2$. Since $\pi$ is $G$-equivariant, it induces a pair of maps $\Omega^{2}(M)^{G} \stackrel{\pi^{*}}{\longrightarrow} \Omega^{2}(X)^{G}$ and $\left(\mathfrak{g}^{*} \otimes \Omega^{2}(M)\right)^{G} \stackrel{\pi^{*}}{\longrightarrow}\left(\mathfrak{g}^{*} \otimes \Omega^{2}(X)\right)^{G}$ and thus also $\Omega_{G}^{2}(M) \stackrel{\pi^{*}}{\longrightarrow} \Omega_{G}^{2}(X)$. Because the face maps are $G$-equivariant, the alternate sum of their pullbacks induces the pair of maps

$$
\begin{gathered}
\Omega^{2}\left(\Gamma_{p-1}\right)^{G} \stackrel{\partial}{\rightarrow} \Omega^{2}\left(\Gamma_{p}\right)^{G} \\
\left(\mathfrak{g}^{*} \otimes \Omega^{0}\left(\Gamma_{p-1}\right)\right)^{G} \stackrel{\partial}{\rightarrow}\left(\mathfrak{g}^{*} \otimes \Omega^{0}\left(\Gamma_{p}\right)\right)^{G},
\end{gathered}
$$

whose direct sum is the desired map

$$
\Omega_{G}^{2}\left(\Gamma_{p-1}\right) \stackrel{\partial}{\rightarrow} \Omega_{G}^{2}\left(\Gamma_{p}\right) .
$$

Since 10 is the direct sum of the exact sequence $(9)$ with $k=2$ (which is exact) and the cochain complex

$$
\left(\mathfrak{g}^{*} \otimes \Omega^{0}(M)\right)^{G} \stackrel{\pi^{*}}{\longrightarrow}\left(\mathfrak{g}^{*} \otimes \Omega^{0}(X)\right)^{G} \stackrel{\partial}{\rightarrow}\left(\mathfrak{g}^{*} \otimes \Omega^{0}(\Gamma)\right)^{G} \stackrel{\partial}{\rightarrow}\left(\mathfrak{g}^{*} \otimes \Omega^{0}\left(\Gamma_{2}\right)\right)^{G} \stackrel{\partial}{\rightarrow} \cdots,
$$

it suffices to prove that the latter sequence is exact. Let $f$ be an arbitrary element of $\left(\mathfrak{g}^{*} \otimes \Omega^{0}\left(\Gamma_{p}\right)\right)^{G}$, i.e.

$$
f\left(\operatorname{Ad}_{g} \xi\right)\left(x_{1}, x_{2}, \ldots, x_{p}\right)=f(\xi)\left(x_{1} \star g, x_{2} \star g, \ldots, x_{p} \star g\right),
$$

for all $\xi \in \mathfrak{g}, g \in G$ and $\left(x_{1}, \ldots, x_{p}\right) \in \Gamma_{p}$. And assume that $\partial f=0$. Choose a basis $\left(e_{1}, \ldots, e_{n}\right)$ of $\mathfrak{g}$. Then $f\left(e_{i}\right) \in \Omega^{0}\left(\Gamma_{p}\right)$ and $\partial\left(f\left(e_{i}\right)\right)=0$. By (a), there exists $h\left(e_{i}\right) \in \Omega^{0}\left(\Gamma_{p-1}\right)$ such that $\partial\left(h\left(e_{i}\right)\right)=f\left(e_{i}\right)$. We can define $h^{\prime}\left(e_{i}\right) \in\left(\mathfrak{g}^{*} \otimes \Omega^{0}\left(\Gamma_{p-1}\right)\right)^{G}$ by

$$
h^{\prime}\left(e_{i}\right)=\frac{1}{V} \int_{G} R_{g}^{*} h\left(\operatorname{Ad}_{g^{-1}} e_{i}\right) d g .
$$

Clearly, $f\left(e_{i}\right)=\partial\left(h^{\prime}\left(e_{i}\right)\right)$ and thus

$$
f\left(\sum_{i} e_{i} \xi^{i}\right)=\partial\left(\sum_{i} h^{\prime}\left(e_{i}\right) \xi^{i}\right)
$$

where $\sum_{i} h^{\prime}\left(e_{i}\right) \xi^{i} \in\left(\mathfrak{g}^{*} \otimes \Omega^{0}\left(\Gamma_{p-1}\right)\right)^{G}$. 
Proof of Proposition 3.2. (a) Take any connection 1-form $\theta^{\prime} \in \Omega^{1}(\widetilde{\Gamma})$ for the $S^{1}$ principal bundle $p: \widetilde{\Gamma} \rightarrow \Gamma$. Since $G$ is compact, one can always take $\theta^{\prime}$ to be $G$-invariant, i.e. $\theta^{\prime} \in \Omega^{1}(\widetilde{\Gamma})^{G}$. It is simple to see that $\widetilde{\partial} \theta^{\prime}$ must be the pull back of a $G$-invariant 1-form on $\Gamma_{2}$ under $p: \widetilde{\Gamma}_{2} \rightarrow \Gamma_{2}$. That is $\widetilde{\partial} \theta^{\prime}=p^{*} \alpha$, where $\alpha \in \Omega^{1}\left(\Gamma_{2}\right)^{G}$. It follows from $\widetilde{\partial}^{2}=0$ that $\partial \alpha=0$. By Lemma 3.3(b), we have $\alpha=\partial A$ for some $A \in \Omega^{1}(\Gamma)^{G}$. Therefore $\theta=\theta^{\prime}-p^{*} A$ is an equivariant connection.

Given an equivariant connection $\theta,(6)$ implies that $\partial \operatorname{curv}_{G}(\theta)=0$ since $\widetilde{\partial} \theta=0$. By Lemma 3.3 (c), there exists $B_{G} \in \Omega_{G}^{2}(X)$ such that $\operatorname{curv}_{G}(\theta)=\partial B_{G}$. That is, $B_{G}$ is an equivariant curving.

Assume that $\left(\theta^{\prime}, B_{G}^{\prime}, \eta_{G}\right)$ is another such triple. We have $\theta-\theta^{\prime}=p^{*} \beta$ for some $\beta \in \Omega^{1}(\Gamma)^{G}$. And $\partial \beta=0$. By Lemma 3.3(b), we have $\beta=\partial \gamma$ for some $\gamma \in \Omega^{1}(X)^{G}$. Now

$$
\begin{aligned}
0 & =d_{G} \theta-d_{G} \theta^{\prime}-p^{*} d_{G} \beta \\
& =p^{*}\left(\operatorname{curv}_{G}(\theta)-\operatorname{curv}_{G}\left(\theta^{\prime}\right)-d_{G} \partial \gamma\right) \\
& =p^{*}\left(\partial\left(B_{G}-B_{G}^{\prime}-d_{G} \gamma\right)\right) .
\end{aligned}
$$

Therefore $\partial\left(B_{G}-B_{G}^{\prime}-d_{G} \gamma\right)=0$. Note that $B_{G}-B_{G}^{\prime}-d_{G} \gamma \in \Omega_{G}^{2}(X)$. Hence, by Lemma 3.3(c), there exists $\lambda \in \Omega_{G}^{2}(M)$ such that $B_{G}-B_{G}^{\prime}-d_{G} \gamma=\pi^{*} \lambda$. Applying $d_{G}$ to both sides, we get $d_{G}\left(B_{G}-B_{G}^{\prime}-d_{G} \gamma\right)=\pi^{*} d_{G} \lambda$, which implies that $\eta_{G}-\eta_{G}^{\prime}=d_{G} \lambda$. The conclusion follows.

The class $\left[\eta_{G}\right]$ is called equivariant Dixmier-Douady class by Meinrenken [12].

3.3. Morita equivalence. Recall that two central $S^{1}$-extensions $\tilde{H} \rightarrow H \rightrightarrows N$ and $\tilde{H}^{\prime} \rightarrow H^{\prime} \rightrightarrows N^{\prime}$ are said to be Morita equivalent [1, 17] if there exists a $\tilde{H}$ - $\tilde{H}^{\prime}$-bitorsor $Z$ endowed with a (left) $S^{1}$-action such that

$$
(\lambda r) \cdot z \cdot r^{\prime}=r \cdot(\lambda z) \cdot r^{\prime}=r \cdot z \cdot\left(\lambda r^{\prime}\right)
$$

whenever $\left(\lambda, r, z, r^{\prime}\right) \in S^{1} \times \tilde{H} \times Z \times \tilde{H}^{\prime}$ and the products make sense.

Definition 3.4. Two G-equivariant bundle gerbes $\widetilde{\Gamma} \stackrel{p}{\rightarrow} \Gamma \rightrightarrows X$ and $\widetilde{\Gamma}^{\prime} \stackrel{p^{\prime}}{\longrightarrow} \Gamma^{\prime} \rightrightarrows$ $X^{\prime}$ are Morita equivalent if they are Morita equivalent as central $S^{1}$-extensions, the equivalence bitorsor $Z$ is a $G$-space and

$$
\left(r \cdot z \cdot r^{\prime}\right) \star g=(r \star g) \cdot(z \star g) \cdot\left(r^{\prime} \star g\right), \quad \forall g \in G
$$

whenever $\left(r, z, r^{\prime}\right) \in \widetilde{\Gamma} \times Z \times \widetilde{\Gamma}^{\prime}$ and the products make sense.

A bitorsor satisfying (11) is called a $G$-equivariant bitorsor.

Proposition 3.5. If $\widetilde{\Gamma} \stackrel{p}{\rightarrow} \Gamma \rightrightarrows X$ and $\widetilde{\Gamma}^{\prime} \stackrel{p^{\prime}}{\longrightarrow} \Gamma^{\prime} \rightrightarrows X^{\prime}$ are Morita equivalent $G$ equivariant bundle gerbes with equivalence bitorsor $Z$, the $S^{1}$-action on $Z$ is free and $Z / S^{1}$ is a $G$-equivariant $\Gamma$ - $\Gamma^{\prime}$-bitorsor. Hence $\Gamma \rightrightarrows X$ and $\Gamma^{\prime} \rightrightarrows X^{\prime}$ are Morita equivalent $G$-groupoids. In other words, the $G$-manifolds $M$ and $M^{\prime}$ underlying the bundle gerbes $p$ and $p^{\prime}$ are one and the same manifold. 


\section{Behrend-Xu-Dixmier-Douady classes}

4.1. General theory. In [1] (see also [17), Behrend-Xu developed a general theory of $S^{1}$-gerbes over differentiable stacks in terms of central $S^{1}$-extensions of Lie groupoids. Roughly speaking, an $S^{1}$-gerbe $\widetilde{\mathfrak{X}}$ over a differentiable stack $\mathfrak{X}$ can be thought of as a Morita equivalence class of central $S^{1}$-extensions of Lie groupoids $H \rightrightarrows N$, where $H \rightrightarrows N$ is a presentation of the differentiable stack $\mathfrak{X}$. (One needs to choose a suitable representative amongst all presentations of the differentiable stack $\mathfrak{X}$, for not every presentation of the stack $\mathfrak{X}$ can be extended to a presentation of the stack $\widetilde{\mathfrak{X}}$. See [1].) According to Giraud [8], the $S^{1}$-gerbes over a differentiable stack $\mathfrak{X}$ are classified by the cohomology group $H^{2}\left(\mathfrak{X}, S^{1}\right)$. Hence, there exists a natural map

$$
\text { \{central } \left.S^{1} \text {-extensions of } H \rightrightarrows N\right\} \stackrel{\tau}{\rightarrow} H^{2}\left(\mathfrak{X}, S^{1}\right) \text {. }
$$

Composing $\tau$ with the boundary map $H^{2}\left(\mathfrak{X}, S^{1}\right) \rightarrow H^{3}(\mathfrak{X}, \mathbb{Z})$ associated to the short exact sequence

$$
0 \rightarrow \mathbb{Z} \rightarrow \mathbb{R} \stackrel{\exp }{\longrightarrow} S^{1} \rightarrow 0
$$

we get a map

$$
\left\{\text { central } S^{1} \text {-extensions of } H \rightrightarrows N\right\} \longrightarrow H^{3}(\mathfrak{X}, \mathbb{Z}) \cong H^{3}\left(H_{\bullet}, \mathbb{Z}\right) \text {. }
$$

The image of a central $S^{1}$-extension under the above map is called its DixmierDouady class in [1].

Behrend-Xu also proved that, similarly to the Chern classes of bundles, the DixmierDouady classes of central $S^{1}$-extensions can be computed, up to torsion elements, from connection type data. Recall that a pseudo-connection on a central $S^{1}$-extension $\tilde{H} \stackrel{p}{\rightarrow} H \rightrightarrows N$ is a sum

$$
\theta+\lambda \in \Omega^{1}(\tilde{H}) \oplus \Omega^{2}(N) \subset \Omega_{\mathrm{DR}}^{2}\left(\tilde{H}_{\bullet}\right)
$$

such that $\theta$ is a connection 1-form on the principal $S^{1}$-bundle $\tilde{H} \stackrel{p}{\rightarrow} H$ 1]. Its pseudocurvature

$$
\eta+\omega+\Omega \in \Omega^{1}\left(H_{2}\right) \oplus \Omega^{2}(H) \oplus \Omega^{3}(N) \subset \Omega_{\mathrm{DR}}^{3}\left(H_{\bullet}\right)
$$

is defined by the relation

$$
\tilde{D}(\theta+\lambda)=p^{*}(\eta+\omega+\Omega) .
$$

Theorem 4.1 (1). The pseudo-curvature $\eta+\omega+\Omega$ is a 3-cocycle in $\Omega_{\mathrm{DR}}^{3}\left(H_{\bullet}\right)$. Its cohomology class $[\eta+\omega+\Omega]$ is an integer class in $H_{\mathrm{DR}}^{3}\left(H_{\bullet}\right)$, which is independent of the choice of pseudo-connections. Under the canonical homomorphism $H^{3}\left(H_{\bullet}, \mathbb{Z}\right) \rightarrow$ $H_{D R}^{3}\left(H_{\bullet}\right)$, the Dixmier-Douady class of $\tilde{H} \stackrel{p}{\rightarrow} H \rightrightarrows N$ maps to $[\eta+\omega+\Omega]$.

The de Rham class $[\eta+\omega+\Omega] \in H_{\mathrm{DR}}^{3}\left(H_{\bullet}\right)$ will be called Behrend-Xu-DixmierDouady class.

4.2. An $S^{1}$-gerbe over $[M / G]$. There is a natural correspondence between equivariant bundle gerbes over a $G$-manifold $M$ in the sense of Murray and Meinrenken and $S^{1}$-gerbes over the stack $[M / G]$ in the sense of Behrend-Xu [1, 16, 17].

The quotient stack $[M / G]$ can be presented by the transformation groupoid $M \rtimes$ $G \rightrightarrows M$, where $t(x, g)=x g, s(x, g)=x$ and

$$
(x, g) \cdot(y, h)=(x, g h), \quad \text { when } y=x \star g .
$$


Adopting the Behrend-Xu perspective, we note that an $S^{1}$-gerbe over the stack $[M / G]$ can always be presented by a central $S^{1}$-extension $\tilde{H} \rightarrow H \rightrightarrows N$ of a Lie groupoid $H \rightrightarrows N$ Morita equivalent to $M \rtimes G \rightrightarrows M$.

Now consider, as in Section 3 , a $G$-equivariant bundle gerbe $\widetilde{\Gamma} \stackrel{p}{\rightarrow} \Gamma \rightrightarrows X$, where $X \stackrel{\pi}{\longrightarrow} M$ is a $G$-equivariant surjective submersion and $\Gamma=X \times_{M} X$. Since $\Gamma \rightrightarrows X$ (resp. $\widetilde{\Gamma} \rightrightarrows X$ ) is a $G$-groupoid, we can form the transformation groupoid $\Gamma \rtimes G \rightrightarrows X$ (resp. $\widetilde{\Gamma} \rtimes G \rightrightarrows X)$.

If $\Gamma_{\bullet}=\left(\Gamma_{1} \rightrightarrows \Gamma_{0}\right)$ is a $G$-groupoid, its transformation groupoid $\Gamma_{\bullet}^{\rtimes}=\left(\Gamma_{1} \rtimes G \rightrightarrows\right.$ $\left.\Gamma_{0}\right)$ is the groupoid whose source map is $s^{\rtimes}:(\gamma, g) \mapsto s(\gamma)$, whose target map is $t^{\rtimes}:(\gamma, g) \mapsto t(\gamma) \star g$ and whose multiplication is given by

$$
\left(\gamma_{1}, g_{1}\right) \cdot\left(\gamma_{2}, g_{2}\right)=\left(\gamma_{1} \cdot\left(\gamma_{2} \star g_{1}^{-1}\right), g_{1} g_{2}\right),
$$

for any $\gamma_{1}, \gamma_{2} \in \Gamma_{1}$ and $g_{1}, g_{2} \in G$ such that $t\left(\gamma_{1}\right) \star g_{1}=s\left(\gamma_{2}\right)$.

Lemma 4.2. (a) The groupoids $\Gamma \rtimes G \rightrightarrows X$ and $M \rtimes G \rightrightarrows M$ are Morita equivalent.

(b) $\operatorname{Set}_{G}(\widetilde{\gamma}, g)=(p(\widetilde{\gamma}), g)$. Then $\widetilde{\Gamma} \rtimes G \stackrel{p_{G}}{\longrightarrow} \Gamma \rtimes G \rightrightarrows X$ is a central $S^{1}$-extension of Lie groupoids.

Proof. (a) Since $\Gamma \rightrightarrows X$ is Morita equivalent to the trivial groupoid $M \rightrightarrows M$ and the Morita morphism mapping $\Gamma \rightrightarrows X$ to $M \rightrightarrows M$ is $G$-equivariant, it follows that the morphism

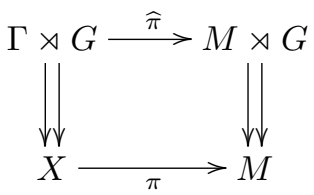

defined by

$$
\widehat{\pi}(x, y, g)=(\pi(y), g), \quad \forall(x, y) \in \Gamma=X \times_{M} X
$$

is a Morita morphism of Lie groupoids.

(b) This follows immediately from the definition of $G$-equivariant central $S^{1}$-extension.

Hence, the extension $\widetilde{\Gamma} \rtimes G \stackrel{p_{G}}{\longrightarrow} \Gamma \rtimes G \rightrightarrows X$ induces an $S^{1}$-gerbe over the quotient stack $[M / G]$ in the sense of Behrend-Xu.

We now compute the Behrend-Xu-Dixmier-Douady class of the central $S^{1}$-extension $\widetilde{\Gamma} \rtimes G \stackrel{p_{G}}{\longrightarrow} \Gamma \rtimes G \rightrightarrows X$. Note that we have the following commutative diagram

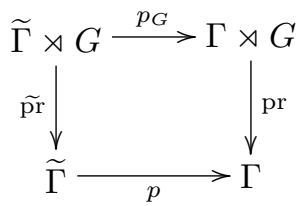

where $\widetilde{p r}$ and pr are mere projection maps rather than groupoid morphisms.

By $\partial^{\rtimes}$ and $\widetilde{\partial}^{\rtimes}$, we denote the coboundary operators associated to the Lie groupoids $\Gamma \rtimes G \rightrightarrows X$ and $\widetilde{\Gamma} \rtimes G \rightrightarrows X$, respectively, as in (1). 
Lemma 4.3. Assume that $\theta \in \Omega^{1}(\widetilde{\Gamma})$ is a G-equivariant connection for the $G$ equivariant bundle gerbe $\widetilde{\Gamma} \stackrel{p}{\rightarrow} \Gamma \rightrightarrows X$. Then $\Theta:=\widetilde{\operatorname{pr}}^{*} \theta \in \Omega^{1}(\widetilde{\Gamma} \rtimes G)$ is a connection 1-form for the principal $S^{1}$-bundle $p_{G}: \widetilde{\Gamma} \rtimes G \rightarrow \Gamma \rtimes G$, hence a pseudo-connection for the central $S^{1}$-extension $\widetilde{\Gamma} \rtimes G \stackrel{p_{G}}{\longrightarrow} \Gamma \rtimes G \rightrightarrows X$. One has

$$
\widetilde{\partial}^{\rtimes} \Theta=p_{G}^{*} \zeta,
$$

where $\zeta \in \Omega^{1}\left((\Gamma \rtimes G)_{2}\right)$ is defined by

$$
\left.\left(p_{G}^{*} \zeta\right)\left(\left(v_{\widetilde{x}}, L_{g *} \xi\right),\left(w_{\widetilde{y}}, L_{h *} \eta\right)\right)=\widehat{\xi}_{\widetilde{y}}\right\lrcorner \theta,
$$

for any $\xi, \eta \in \mathfrak{g}, g, h \in G, v_{\widetilde{x}} \in T_{\widetilde{x}} \widetilde{\Gamma}$ and $w_{\widetilde{y}} \in T_{\widetilde{y}} \widetilde{\Gamma}$ such that $t^{\rtimes}\left(v_{\widetilde{x}}, L_{g *} \xi\right)=$ $s^{\rtimes}\left(w_{\widetilde{y}}, L_{h *} \eta\right)$.

Proof. Let $t \mapsto g_{t}$ and $t \mapsto h_{t}$ be paths in $G$ originating from $g$ and $h$ respectively and determining two vectors $\xi$ and $\eta$ of $\mathfrak{g}$ by the relations $L_{g *} \xi=\left.\frac{d}{d t} g_{t}\right|_{0}$ and $L_{h *} \eta=\left.\frac{d}{d t} h_{t}\right|_{0}$. Similarly, let $t \mapsto \widetilde{x}_{t}$ and $t \mapsto \widetilde{y}_{t}$ be smooth paths in $\widetilde{\Gamma}$ originating from $\widetilde{x}$ and $\widetilde{y}$, respectively, with $\left.\frac{d}{d t} \widetilde{x}_{t}\right|_{0}=v_{\widetilde{x}}$ and $\left.\frac{d}{d t} \widetilde{y}_{t}\right|_{0}=w_{\widetilde{y}}$ and such that, at any time $t$, the target of $\widetilde{x}_{t} \star g_{t}$ coincides with the source of $\widetilde{y}_{t}$. Then

$$
\begin{aligned}
& \left(\widetilde{\partial}^{\rtimes} \Theta\right)\left(\left.\frac{d}{d t}\left(\widetilde{x}_{t}, g_{t}\right)\right|_{0},\left.\frac{d}{d t}\left(\widetilde{y}_{t}, h_{t}\right)\right|_{0}\right) \\
& =\Theta\left(\left.\frac{d}{d t}\left(\widetilde{y}_{t}, h_{t}\right)\right|_{0}\right)-\Theta\left(\left.\frac{d}{d t}\left(\widetilde{x}_{t} \cdot\left(\widetilde{y}_{t} \star g_{t}^{-1}\right), g_{t} h_{t}\right)\right|_{0}\right) \\
& +\Theta\left(\left.\frac{d}{d t}\left(\widetilde{x}_{t}, g_{t}\right)\right|_{0}\right) \\
& =\theta\left(\left.\frac{d}{d t} \widetilde{y}_{t}\right|_{0}\right)-\theta\left(\left.\frac{d}{d t}\left(\widetilde{x}_{t} \cdot\left(\widetilde{y}_{t} \star g_{t}^{-1}\right)\right)\right|_{0}\right)+\theta\left(\left.\frac{d}{d t} \widetilde{x}_{t}\right|_{0}\right) \quad \text { since } \Theta=\operatorname{pr}^{*} \theta \\
& =\theta\left(\left.\frac{d}{d t} \widetilde{y}_{t}\right|_{0}\right)-\theta\left(\left.\frac{d}{d t} \widetilde{x}_{t}\right|_{0}\right)-\theta\left(\left.\frac{d}{d t}\left(\widetilde{y}_{t} \star g_{t}^{-1}\right)\right|_{0}\right)+\theta\left(\left.\frac{d}{d t} \widetilde{x}_{t}\right|_{0}\right) \quad \text { since } \widetilde{\partial} \theta=0 \\
& =\theta\left(\left.\frac{d}{d t} \widetilde{y}_{t}\right|_{0}\right)-\theta\left(\left.\frac{d}{d t}\left(\widetilde{y}_{t} \star g^{-1}\right)\right|_{0}\right)-\theta\left(\left.\frac{d}{d t}\left(\widetilde{y} \star g_{t}^{-1}\right)\right|_{0}\right) \\
& \left.=-\left.\theta\left(\frac{d}{d t} \widetilde{y} \star\left(g e^{t \xi}\right)^{-1}\right)\right|_{0}\right) \\
& =\theta\left(\widehat{\xi}_{\widetilde{y}} \star g^{-1}\right) \\
& =\theta\left(\widehat{\xi}_{\widetilde{y}}\right) \\
& \text { since } \theta \text { is } G \text {-invariant } \\
& \text { since } \theta \text { is } G \text {-invariant. }
\end{aligned}
$$

The result follows.

Proposition 4.4. Let $\theta \in \Omega^{1}(\widetilde{\Gamma})$ be a $G$-equivariant connection on a $G$-equivariant bundle gerbe $\widetilde{\Gamma} \stackrel{p}{\rightarrow} \Gamma \rightrightarrows X$ over a $G$-manifold $M$. Then $\Theta:=\widetilde{p r}^{*} \theta \in \Omega^{1}(\widetilde{\Gamma} \rtimes G)$ is a pseudo-connection for the central $S^{1}$-extension $\widetilde{\Gamma} \rtimes G \stackrel{p_{G}}{\longrightarrow} \Gamma \rtimes G \rightrightarrows X$. Its pseudocurvature is $\zeta-\operatorname{pr}^{*} \omega \in Z^{3}((\Gamma \times G)$. $)$, where $\zeta$ is given by (13) and $\omega$ is characterized by $d \theta=p^{*} \omega$. Hence the Behrend-Xu-Dixmier-Douady class is $\left[\zeta-\operatorname{pr}^{*} \omega\right] \in H_{\mathrm{DR}}^{3}((\Gamma \rtimes$ $G)$.).

Proof. Since

$$
d \Theta=d \widetilde{\operatorname{pr}}^{*} \theta=\widetilde{\operatorname{pr}}^{*} d \theta=\widetilde{\operatorname{pr}}^{*} p^{*} \omega=p_{G}^{*} \operatorname{pr}^{*} \omega,
$$

the associated pseudo-curvature is

$$
\widetilde{D}^{\rtimes} \Theta=\widetilde{\partial}^{\rtimes} \Theta-d \Theta=p_{G}^{*}\left(\zeta-\operatorname{pr}^{*} \omega\right) .
$$


Remark 4.5. From Lemma 4.3 , we see that $\widetilde{\partial}^{\rtimes} \Theta$ vanishes if, and only if, $\widetilde{\partial} \theta=0$ and $\theta$ is basic with respect to the $G$-action. In this case, $\Theta$ is a connection for the central $S^{1}$-extension $\widetilde{\Gamma} \rtimes G \stackrel{p_{G}}{\longrightarrow} \Gamma \rtimes G \rightrightarrows X$ in the sense of Behrend-Xu [1]. See [16 for details.

\section{Linking Murray-Meinrenken to Behrend-Xu}

5.1. The BCWZ morphism. In order to compare Meinrenken's equivariant DixmierDouady class with the Behrend-Xu-Dixmier-Douady class, we need an explicit formula relating the Cartan and simplicial models of equivariant cohomology in degree 3 . The following result can be found in [3] (though the group acts from the left in [3]).

Proposition 5.1 (Proposition 6.10 in [3]). Let $N$ be a manifold on which a Lie group $G$ acts from the right. Consider the map

$$
\psi: \Omega_{G}^{3}(N) \rightarrow \Omega_{\mathrm{DR}}^{3}((N \rtimes G) \text {. })
$$

mapping $\alpha \in \Omega^{3}(N)$ to itself $(\psi(\alpha)=\alpha)$ and $\eta \in\left(\mathfrak{g}^{*} \otimes \Omega^{1}(N)\right)^{G}$ to the 2-form $\psi(\eta) \in \Omega^{2}(N \rtimes G)$ defined by

(14) $\psi(\eta)\left(\left(v_{1}, L_{g *} \xi_{1}\right),\left(v_{2}, L_{g *} \xi_{2}\right)\right)=\eta\left(\xi_{2}\right)\left(v_{1} \star g\right)-\eta\left(\xi_{1}\right)\left(v_{2} \star g\right)+\eta\left(\xi_{2}\right)\left(\left.\widehat{\xi_{1}}\right|_{x \star g}\right)$,

for all $v_{1}, v_{2} \in T_{x} N, \xi_{1}, \xi_{2} \in \mathfrak{g}$ and $g \in G$. The map $\psi$ injects $Z^{3}(N)^{G}$ into $Z_{\mathrm{DR}}^{3}((N \rtimes$ $G)$.$) . Moreover,$

$$
\psi\left(Z^{3}(N)^{G}\right)=Z_{\mathrm{DR}}^{3}((N \rtimes G) .) \cap\left(\Omega^{3}(M) \oplus \Omega^{2}(N \rtimes G)\right) .
$$

Remark 5.2. One can check that the R.H.S. of (14) does indeed change sign when the indices 1 and 2 are permuted.

From (14), one easily deduces that, if $\sigma: N_{1} \rightarrow N_{2}$ is a $G$-equivariant map between two $G$-manifolds $N_{1}$ and $N_{2}$, then

$$
\psi\left(\sigma^{*} \eta\right)=(\sigma \times 1)^{*} \psi(\eta), \quad \forall \eta \in\left(\mathfrak{g}^{*} \otimes \Omega^{1}\left(N_{2}\right)\right)^{G} .
$$

The following lemma will be needed later on.

Lemma 5.3. Given $f \in\left(\mathfrak{g}^{*} \otimes \Omega^{0}(N)\right)^{G}$, then

$$
\psi(d f)=d \lambda,
$$

where $\lambda \in \Omega^{1}(N \times G)$ is defined by the relation

$$
\lambda\left(v_{x}, L_{g *} \xi\right)=f\left(\operatorname{Ad}_{g} \xi\right)(x), \quad \forall v_{x} \in T_{x} N, \xi \in \mathfrak{g}, g \in G .
$$

Proof. Since $f$ is $G$-equivariant, we have

$$
f\left(\operatorname{Ad}_{g} \xi\right)(x)=f(\xi)(x \star g) .
$$

Take $g \in G, \xi_{1}, \xi_{2} \in \mathfrak{g}$ and $v_{1}, v_{2} \in T_{x} N$. Let $t \mapsto x_{1}(t)$ and $t \mapsto x_{2}(t)$ be two paths in $N$ originating from the same point $x$ such that $v_{1}=\left.\frac{d}{d t} x_{1}(t)\right|_{0}$ and $v_{2}=\left.\frac{d}{d t} x_{2}(t)\right|_{0}$. 
From 14, we get

$$
\begin{aligned}
& \psi(d f)\left(\left(v_{1}, L_{g *} \xi_{1}\right),\left(v_{2}, L_{g *} \xi_{2}\right)\right) \\
= & \psi(d f)\left(\left.\frac{d}{d t}\left(x_{1}(t), g e^{t \xi_{1}}\right)\right|_{0},\left.\frac{d}{d t}\left(x_{2}(t), g e^{t \xi_{2}}\right)\right|_{0}\right) \\
= & \left.\frac{d}{d t} f\left(\xi_{2}\right)\left(x_{1}(t) \star g\right)\right|_{0}-\left.\frac{d}{d t} f\left(\xi_{1}\right)\left(x_{2}(t) \star g\right)\right|_{0}+\left.\frac{d}{d t} f\left(\xi_{2}\right)\left(x \star\left(g e^{t \xi_{1}}\right)\right)\right|_{0} \\
= & \left.\frac{d}{d t} f\left(\operatorname{Ad}_{g} \xi_{2}\right)\left(x_{1}(t)\right)\right|_{0}-\left.\frac{d}{d t} f\left(\operatorname{Ad}_{g} \xi_{1}\right)\left(x_{2}(t)\right)\right|_{0}+\left.\frac{d}{d t} f\left(\operatorname{Ad}_{g} e^{t \operatorname{ad}_{\xi_{1}} \xi_{2}}\right)(x)\right|_{0} \text { by 17). }
\end{aligned}
$$

But $f$ is linear in $\mathfrak{g}$. Hence the last term is equal to $f\left(\operatorname{Ad}_{g}\left[\xi_{1}, \xi_{2}\right]\right)(x)$.

On the other hand, letting $\overleftarrow{\xi_{1}}$ and $\overleftarrow{\xi_{2}}$ be the left invariant vector fields on $G$ corresponding to $\xi_{1}$ and $\xi_{2}$, respectively, and choosing two vector fields $X_{1}$ and $X_{2}$ on $N$ such that $\left.X_{1}\right|_{x}=v_{1}$ and $\left.X_{2}\right|_{x}=v_{2}$, we obtain

$$
\begin{aligned}
& (d \lambda)\left(\left(v_{1}, L_{g *} \xi_{1}\right),\left(v_{2}, L_{g *} \xi_{2}\right)\right) \\
= & \left.\left(v_{1}, L_{g *} \xi_{1}\right) \lambda\left(X_{2}, \overleftarrow{\xi_{2}}\right)-\left(v_{2}, L_{g *} \xi_{2}\right) \lambda\left(X_{1}, \overleftarrow{\xi_{1}}\right)-\lambda\left(\left[X_{1}, X_{2}\right]_{x}, \overleftarrow{\xi_{1}}, \overleftarrow{\xi_{2}}\right]_{g}\right) \\
= & \left.\frac{d}{d t} f\left(\operatorname{Ad}_{g e^{t \xi_{1}}} \xi_{2}\right)\left(x_{1}(t)\right)\right|_{0}-\left.\frac{d}{d t} f\left(\operatorname{Ad}_{g e^{t \xi_{2}}} \xi_{1}\right)\left(x_{2}(t)\right)\right|_{0}-f\left(\operatorname{Ad}_{g}\left[\xi_{1}, \xi_{2}\right]\right)(x) \\
= & \left.\frac{d}{d t} f\left(\operatorname{Ad}_{g e^{t \xi_{1}}} \xi_{2}\right)(x)\right|_{0}+\left.\frac{d}{d t} f\left(\operatorname{Ad}_{g} \xi_{2}\right)\left(x_{1}(t)\right)\right|_{0}-\left.\frac{d}{d t} f\left(\operatorname{Ad}_{g e^{t \xi_{2}}} \xi_{1}\right)(x)\right|_{0} \\
& -\left.\frac{d}{d t} f\left(\operatorname{Ad}_{g} \xi_{1}\right)\left(x_{2}(t)\right)\right|_{0}-f\left(\operatorname{Ad}_{g}\left[\xi_{1}, \xi_{2}\right]\right)(x) \\
= & \left.\frac{d}{d t} f\left(\operatorname{Ad}_{g} \xi_{2}\right)\left(x_{1}(t)\right)\right|_{0}-\left.\frac{d}{d t} f\left(\operatorname{Ad}_{g} \xi_{1}\right)\left(x_{2}(t)\right)\right|_{0}+f\left(\operatorname{Ad}_{g}\left[\xi_{1}, \xi_{2}\right]\right)(x) .
\end{aligned}
$$

The result follows.

Lemma 5.4. Given $B \in \Omega^{2}(N)^{G}$, define $Q \in\left(\mathfrak{g}^{*} \otimes \Omega^{1}(N)\right)^{G}$ by $\left.Q(\xi)=\widehat{\xi}\right\lrcorner B$, $\forall \xi \in \mathfrak{g}$. Then $\psi(Q)=-\partial^{\times} B$, where $\partial^{\times}: \Omega^{2}(N) \rightarrow \Omega^{2}(N \rtimes G)$ is the coboundary operator $\partial^{\times}=t^{*}-s^{*}$ associated to the transformation groupoid $N \rtimes G \rightrightarrows N$.

Proof. Note that for any $v_{x} \in T_{x} N$ and $\xi \in \mathfrak{g}$,

$$
s_{*}\left(v_{x}, L_{g *} \xi\right)=v_{x} \quad \text { and } \quad t_{*}\left(v_{x}, L_{g *} \xi\right)=v_{x} \star g+\left.\widehat{\xi}\right|_{x \star g} .
$$

Thus, using the $G$-invariance of $B$, we obtain, for any $v_{1}, v_{2} \in T_{x} N, \xi_{1}, \xi_{2} \in \mathfrak{g}$ and $g \in G$,

$$
\begin{aligned}
& \left(\partial^{\times} B\right)\left(\left(v_{1}, L_{g *} \xi_{1}\right),\left(v_{2}, L_{g *} \xi_{2}\right)\right) \\
= & B\left(t_{*}\left(v_{1}, L_{g *} \xi_{1}\right), t_{*}\left(v_{2}, L_{g *} \xi_{2}\right)\right)-B\left(s_{*}\left(v_{1}, L_{g *} \xi_{1}\right), s_{*}\left(v_{2}, L_{g *} \xi_{2}\right)\right) \\
= & B\left(\left.\widehat{\xi_{1}}\right|_{x \star g}, v_{2} \star g\right)+B\left(v_{1} \star g,\left.\widehat{\xi_{2}}\right|_{x \star g}\right)+B\left(\left.\widehat{\xi_{1}}\right|_{x \star g},\left.\widehat{\xi_{2}}\right|_{x \star g}\right) .
\end{aligned}
$$

On the other hand, we have

$$
\begin{aligned}
& \psi(Q)\left(\left(v_{1}, L_{g *} \xi_{1}\right),\left(v_{2}, L_{g *} \xi_{2}\right)\right) \\
= & Q\left(\xi_{2}\right)\left(v_{1} \star g\right)-Q\left(\xi_{1}\right)\left(v_{2} \star g\right)+Q\left(\xi_{2}\right)\left(\left.\widehat{\xi_{1}}\right|_{x \star g}\right) \\
= & B\left(\left.\widehat{\xi_{2}}\right|_{x \star g}, v_{1} \star g\right)-B\left(\left.\widehat{\xi_{1}}\right|_{x \star g}, v_{2} \star g\right)+B\left(\left.\widehat{\xi_{2}}\right|_{x \star g},\left.\widehat{\xi_{1}}\right|_{x \star g}\right) .
\end{aligned}
$$

The conclusion thus follows.

As an immediate consequence, we have

Corollary 5.5. The BCWZ map $\psi$ of Proposition 5.1 maps the exact equivariant 3-forms $B_{G}^{3}(N)$ to the coboundaries $B_{\mathrm{DR}}^{3}((N \rtimes G)$ •). Therefore $\psi$ induces an isomorphism in cohomology: $H_{G}^{3}(N) \stackrel{\cong}{\longrightarrow} H_{\mathrm{DR}}^{3}((N \rtimes G)$.). 
Proof. The 1-form $\lambda \in \Omega^{1}(N \times G)$ defined in Lemma 5.3 satisfies

$$
\partial^{\times} \lambda=0 .
$$

Indeed, since $t_{*}\left(v_{x}, L_{g *} \xi\right)=s_{*}\left(w_{y}, L_{h *} \eta\right)$ if, and only if, $y=x \star g$ and $w_{y}=v_{x} \star g+\widehat{\xi}_{x \star g}$, making use of the linearity in $\mathfrak{g}$ and the $G$-equivariance of $f$ we obtain

$$
\begin{aligned}
& \partial^{\times} \lambda\left(\left(v_{x}, L_{g *} \xi\right),\left(w_{y}, L_{h *} \eta\right)\right) \\
= & \lambda\left(w_{y}, L_{h *} \eta\right)-\lambda\left(v_{x}, L_{g h *}\left(\operatorname{Ad}_{h^{-1}} \xi+\eta\right)\right)+\lambda\left(v_{x}, L_{g *} \xi\right) \\
= & f\left(\operatorname{Ad}_{h} \eta\right)(y)-f\left(\operatorname{Ad}_{g h}\left(\operatorname{Ad}_{h^{-1}} \xi+\eta\right)\right)(x)+f\left(\operatorname{Ad}_{g} \xi\right)(x) \\
= & f\left(\operatorname{Ad}_{h} \eta\right)(x \star g)-f\left(\operatorname{Ad}_{g}\left(\operatorname{Ad}_{h} \eta\right)\right)(x) \\
= & 0 .
\end{aligned}
$$

Since

$$
\Omega_{G}^{2}(N)=\Omega^{2}(N)^{G} \oplus\left(\mathfrak{g}^{*} \otimes \Omega^{0}(N)\right)^{G},
$$

any element of $B_{G}^{3}(N)=d_{G}\left(\Omega_{G}^{2}(N)\right)$ can be written as $d_{G}(B+f)$ for some $B \in$ $\Omega^{2}(N)^{G}$ and $f \in\left(\mathfrak{g}^{*} \otimes \Omega^{0}(N)\right)^{G}$. By definition, $d_{G} B=d B-Q$, where $Q$ is defined as in Lemma 5.4, and $d_{G} f=d f$. Therefore,

$$
\begin{aligned}
\psi\left(d_{G}(B+f)\right) & =\psi(d B-Q+d f) & & \\
& =d B+\partial^{\times} B+d \lambda & & \text { by Lemma } 5.3 \text { and } 5.4 \\
& =D^{\times}(B+\lambda) & & \text { by } 18 .
\end{aligned}
$$

Thus $\psi$ not only maps closed equivariant 3 -forms to cocycles of $\Omega_{\mathrm{DR}}^{3}((N \rtimes G)$.) (see Proposition 5.1) but also exact equivariant 3 -forms to coboundaries of $\Omega_{\mathrm{DR}}^{3}((N \rtimes$ $G)$.). Hence $\psi$ induces a homomorphism $H_{G}^{3}(N) \rightarrow H_{\mathrm{DR}}^{3}((N \rtimes G)$ •) in cohomology. Actually, the latter is an isomorphism, for $\psi$ is injective on the level of cocycles (according to Proposition 5.1) and any 3-cocycle in $\Omega_{\mathrm{DR}}^{3}((N \rtimes G)$.$) is cohomologous$ to a 3-cocycle of the form $\alpha+\beta$, where $\alpha \in \Omega^{3}(N)$ and $\beta \in \Omega^{2}(N \times G)$. Indeed, since $N \rtimes G \rightrightarrows N$ is a proper Lie groupoid, the sequence

$$
\Omega^{0}\left((N \rtimes G)_{2}\right) \stackrel{\partial^{\times}}{\longrightarrow} \Omega^{0}\left((N \rtimes G)_{3}\right) \stackrel{\partial^{\times}}{\longrightarrow} \Omega^{0}\left((N \rtimes G)_{4}\right)
$$

is exact [5, Proposition 1] and, moreover,

$$
\Omega^{1}\left((N \rtimes G)_{1}\right) \stackrel{\partial^{\times}}{\longrightarrow} \Omega^{1}\left((N \rtimes G)_{2}\right) \stackrel{\partial^{\times}}{\longrightarrow} \Omega^{1}\left((N \rtimes G)_{3}\right)
$$

is also exact [16, Lemma 1.5].

5.2. Main theorem. The Morita morphism

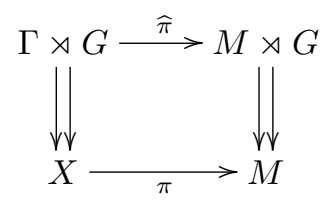

as in 12 induces a map of cochain complexes

$$
\Omega_{\mathrm{DR}}^{k}((M \rtimes G) .) \stackrel{\widehat{\pi}^{*}}{\longrightarrow} \Omega_{\mathrm{DR}}^{k}((\Gamma \rtimes G) .)
$$


which gives an isomorphism in cohomology:

$$
H_{\mathrm{DR}}^{k}((M \rtimes G) .) \stackrel{\cong}{\longrightarrow} H_{\mathrm{DR}}^{k}((\Gamma \rtimes G) .) .
$$

By the symbol $\mu$, we will denote both the composition

$$
Z_{G}^{3}(M) \stackrel{\psi}{\longrightarrow} Z_{\mathrm{DR}}^{3}((M \rtimes G) .) \stackrel{\widehat{\pi}^{*}}{\longrightarrow} Z_{\mathrm{DR}}^{3}\left((\Gamma \rtimes G)_{\bullet}\right)
$$

and the induced isomorphism

$$
H_{G}^{3}(M) \stackrel{\cong}{\longrightarrow} H_{\mathrm{DR}}^{3}((\Gamma \rtimes G) .)
$$

in cohomology.

The main theorem can be stated as follows.

Theorem 5.6. Let $\widetilde{\Gamma} \stackrel{p}{\rightarrow} \Gamma \rightrightarrows X$ be a G-equivariant bundle gerbe over a $G$-manifold $M$ with equivariant connection $\theta$, equivariant curving $B_{G}$ and equivariant 3-curvature $\eta_{G}$. Then

$$
\mu\left[\eta_{G}\right]=\left[\zeta-\operatorname{pr}^{*} \omega\right]
$$

in $H_{\mathrm{DR}}^{3}((\Gamma \rtimes G)$.$) . In other words, the isomorphism \mu$ maps Meinrenken's equivariant Dixmier-Douady class to the Behrend-Xu-Dixmier-Douady class.

Given two Morita equivalent $G$-equivariant bundle gerbes $\widetilde{\Gamma} \stackrel{p}{\rightarrow} \Gamma \rightrightarrows X$ and $\widetilde{\Gamma}^{\prime} \stackrel{p^{\prime}}{\longrightarrow}$ $\Gamma^{\prime} \rightrightarrows X^{\prime}$ over a $G$-manifold $M$, it is simple to see that $\widetilde{\Gamma} \rtimes G \stackrel{p_{G}}{\longrightarrow} \Gamma \rtimes G \rightrightarrows X$ and $\widetilde{\Gamma}^{\prime} \rtimes G \stackrel{p_{G}^{\prime}}{\longrightarrow} \Gamma^{\prime} \rtimes G \rightrightarrows X^{\prime}$ are Morita equivalent central $S^{1}$-extensions. Hence they have isomorphic Behrend-Xu-Dixmier-Douady classes according to [1. As a consequence of Theorem 5.6, we have

Corollary 5.7. Morita equivalent $G$-equivariant bundle gerbes have isomorphic equivariant Dixmier-Douady classes in Meinrenken's sense.

Remark 5.8. The above corollary asserts the existence of a map

$$
\left\{\begin{array}{l}
\text { Morita equivalence classes of } \\
G \text {-equivariant bundle gerbes } \\
\text { over } M
\end{array}\right\} \rightarrow H_{G}^{3}(M, \mathbb{Z}),
$$

which is easily seen to be injective. It is not clear though if this map is surjective since requiring that a gerbe be $G$-equivariant may seem too strong (see Remark 2.8 in $[12$ ). Recently, however, Tu-Xu proved that the above map is indeed also surjective [16].

5.3. Proof of the main theorem. First of all, let us wrap off the conditions defining equivariant connections, equivariant curvings and equivariant 3 -curvatures. Since $\Omega_{G}^{2}(\Gamma)=\Omega^{2}(\Gamma)^{G} \oplus\left(\mathfrak{g}^{*} \otimes \Omega^{0}(\Gamma)\right)^{G}$, the equivariant curvature decomposes as

$$
\operatorname{curv}_{G}(\theta)=\omega+\phi,
$$

where $\omega \in \Omega^{2}(\Gamma)^{G}$ and $\phi \in\left(\mathfrak{g}^{*} \otimes \Omega^{0}(\Gamma)\right)^{G}$. From (6), we obtain

$$
d \theta-\widehat{\xi}\lrcorner \theta=p^{*} \omega+\left(p^{*} \phi\right)(\xi), \quad \forall \xi \in \mathfrak{g},
$$


which is equivalent to the pair of equations

$$
\begin{gathered}
d \theta=p^{*} \omega \\
-\widehat{\xi}\lrcorner \theta=\left(p^{*} \phi\right)(\xi), \quad \forall \xi \in \mathfrak{g}
\end{gathered}
$$

Since $\Omega_{G}^{2}(X)=\Omega^{2}(X)^{G} \oplus\left(\mathfrak{g}^{*} \otimes \Omega^{0}(X)\right)^{G}$, the curving decomposes as

$$
B_{G}=B+f,
$$

where $B \in \Omega^{2}(X)^{G}$ and $f \in\left(\mathfrak{g}^{*} \otimes \Omega^{0}(X)\right)^{G}$. From (5), we obtain

$$
\omega+\phi=\partial(B+f)
$$

or, equivalently,

$$
\begin{aligned}
& \omega=\partial B \\
& \phi=\partial f .
\end{aligned}
$$

Hence $\widetilde{\partial} f=p^{*} \partial f=p^{*} \phi$ and

$$
\left.(\widetilde{\partial} f)(\xi)=\left(p^{*} \phi\right)(\xi)=-\widehat{\xi}\right\lrcorner \theta .
$$

Since $\Omega_{G}^{3}(M)=\Omega^{3}(M)^{G} \oplus\left(\mathfrak{g}^{*} \otimes \Omega^{1}(M)\right)^{G}$, the 3-curvature decomposes as

$$
\eta_{G}=\alpha+\eta,
$$

where $\alpha \in \Omega^{3}(M)^{G}$ and $\eta \in\left(\mathfrak{g}^{*} \otimes \Omega^{1}(M)\right)^{G}$. From (7), we obtain

$$
\left.\pi^{*}(\alpha+\eta)=d_{G}(B+f)=d B-\widehat{\xi}\right\lrcorner B+d f
$$

and it thus follows that

$$
\begin{gathered}
\pi^{*} \alpha=d B \\
\left.\pi^{*} \eta=d f-\widehat{\xi}\right\lrcorner B .
\end{gathered}
$$

We will need a few lemmas.

Lemma 5.9. We have $\left[\psi\left(t^{*} d f\right)-\zeta\right]=0$ in $H_{\mathrm{DR}}^{3}((\Gamma \rtimes G)$.).

Proof. Since $t: \Gamma \rightarrow X$ is $G$-equivariant, from (15) and (16), we obtain

$$
\psi\left(t^{*} d f\right)=(t \times \mathrm{id})^{*} \psi(d f)=(t \times \mathrm{id})^{*} d \lambda=d \lambda^{\prime},
$$

where $\lambda^{\prime}=(t \times \text { id })^{*} \lambda \in \Omega^{1}(\Gamma \times G)$. More explicitly, $\forall\left(v_{x}, w_{y}\right) \in T_{(x, y)} \Gamma, \xi \in \mathfrak{g}, g \in G$, we have

$$
\lambda^{\prime}\left(\left(v_{x}, w_{y}\right), L_{g *} \xi\right)=\lambda\left(w_{y}, L_{g *} \xi\right)=f\left(\operatorname{Ad}_{g} \xi\right)(y) .
$$

The multiplication in the groupoid $\Gamma \rtimes G \rightrightarrows X$ is defined by

$$
((x, y), g) \cdot\left(\left(x^{\prime}, y^{\prime}\right), h\right)=\left(\left(x, y^{\prime} \star g^{-1}\right), g h\right),
$$

provided $y \star g=x^{\prime}$, where $(x, y),\left(x^{\prime}, y^{\prime}\right) \in \Gamma\left(\cong X \times_{M} X\right)$ and $g, h \in G$. It thus follows that

$$
\begin{aligned}
& \left(\partial^{\rtimes} \lambda^{\prime}\right)\left(\left(v_{x}, w_{y}, L_{g *} \xi\right),\left(v_{x^{\prime}}^{\prime}, w_{y^{\prime}}^{\prime}, L_{h *} \eta\right)\right) \\
& \quad=\lambda^{\prime}\left(v_{x}, w_{y}, L_{g *} \xi\right)-\lambda^{\prime}\left(\left(v_{x}, w_{y}, L_{g *} \xi\right) \cdot\left(v_{x^{\prime}}^{\prime}, w_{y^{\prime}}^{\prime}, L_{h *} \eta\right)\right)+\lambda^{\prime}\left(v_{x^{\prime}}^{\prime}, w_{y^{\prime}}^{\prime}, L_{h *} \eta\right) .
\end{aligned}
$$

But (24) implies that

$$
\left(v_{x}, w_{y}, L_{g *} \xi\right) \cdot\left(v_{x^{\prime}}^{\prime}, w_{y^{\prime}}^{\prime}, L_{h *} \eta\right)=\left(v_{x}^{\prime \prime}, w_{y^{\prime} \star g^{-1}}^{\prime \prime}, L_{g h *}\left(\operatorname{Ad}_{h^{-1}} \xi+\eta\right)\right),
$$


where $v_{x}^{\prime \prime}$ and $w_{y^{\prime} \star g^{-1}}^{\prime \prime}$ are tangent vectors of $X$ at $x$ and $y^{\prime} \star g^{-1}$ respectively. Hence we get

$$
\begin{aligned}
& \lambda^{\prime}\left(\left(v_{x}, w_{y}, L_{g *} \xi\right) \cdot\left(v_{x^{\prime}}^{\prime}, w_{y^{\prime}}^{\prime}, L_{h *} \eta\right)\right) \\
& =f\left(\operatorname{Ad}_{g h}\left(\operatorname{Ad}_{h^{-1}} \xi+\eta\right)\right)\left(y^{\prime} \star g^{-1}\right) \\
& =f\left(\operatorname{Ad}_{g^{-1}} \operatorname{Ad}_{g h}\left(\operatorname{Ad}_{h^{-1}} \xi+\eta\right)\right)\left(y^{\prime}\right) \quad \text { since } f \text { is } G \text {-equivariant } \\
& =f(\xi)\left(y^{\prime}\right)+f\left(\operatorname{Ad}_{h} \eta\right)\left(y^{\prime}\right) \quad \text { since } f \text { is linear in } \mathfrak{g} \text {. }
\end{aligned}
$$

Thus, we have

$$
\begin{aligned}
& \left(\partial^{\rtimes} \lambda^{\prime}\right)\left(\left(v_{x}, w_{y}, L_{g *} \xi\right),\left(v_{x^{\prime}}^{\prime}, w_{y^{\prime}}^{\prime}, L_{h *} \eta\right)\right) \\
= & f\left(\operatorname{Ad}_{g} \xi\right)(y)-\left(f(\xi)\left(y^{\prime}\right)+f\left(\operatorname{Ad}_{h} \eta\right)\left(y^{\prime}\right)\right)+f\left(\operatorname{Ad}_{h} \eta\right)\left(y^{\prime}\right) \\
= & f\left(\operatorname{Ad}_{g} \xi\right)(y)-f(\xi)\left(y^{\prime}\right) \\
= & f(\xi)(y \star g)-f(\xi)\left(y^{\prime}\right) \\
= & f(\xi)\left(x^{\prime}\right)-f(\xi)\left(y^{\prime}\right) \\
= & -(\partial f)(\xi)\left(x^{\prime}, y^{\prime}\right) .
\end{aligned}
$$

Now, set $z=(x, y)$ and $z^{\prime}=\left(x^{\prime}, y^{\prime}\right) \in \Gamma=X \times_{M} X$ and choose $\widetilde{z}$ and $\widetilde{z}^{\prime} \in \widetilde{\Gamma}$ such that $p(\widetilde{z})=z$ and $p\left(\widetilde{z}^{\prime}\right)=z^{\prime}$. Moreover, take $v_{\widetilde{z}} \in T_{\widetilde{z}} \widetilde{\Gamma}$ and $v_{\widetilde{z}^{\prime}} \in T_{\widetilde{z}^{\prime}} \widetilde{\Gamma}$ such that $p_{G *} v_{\widetilde{z}}=\left(v_{x}, w_{y}\right)$ and $p_{G *} v_{\widetilde{z}^{\prime}}=\left(v_{x^{\prime}}^{\prime}, w_{y^{\prime}}^{\prime}\right)$. Then

$$
\begin{aligned}
& p_{G}^{*}\left(\partial^{\rtimes} \lambda^{\prime}\right)\left(\left(v_{\widetilde{z}}, L_{g *} \xi\right),\left(v_{\widetilde{z}^{\prime}},, L_{h *} \eta\right)\right) \\
= & \left(\partial^{\rtimes} \lambda^{\prime}\right)\left(\left(v_{x}, w_{y}, L_{g *} \xi\right),\left(v_{x^{\prime}}^{\prime}, w_{y^{\prime}}^{\prime}, L_{h *} \eta\right)\right) \\
= & -(\partial f)(\xi)\left(x^{\prime}, y^{\prime}\right) \\
= & -p_{G}^{*}((\partial f)(\xi))\left(\widetilde{z}^{\prime}\right) \\
= & -(\widetilde{\partial} f)(\xi)\left(\widetilde{z}^{\prime}\right) \\
= & \widehat{\xi}_{\widetilde{z}^{\prime}}-\theta \\
= & \left(p_{G}^{*} \zeta\right)\left(\left(v_{\widetilde{z}}, L_{g *} \xi\right),\left(v_{\widetilde{z}^{\prime}}, L_{h *} \eta\right)\right)
\end{aligned}
$$

by 20

by $(13)$.

Hence

$$
\partial^{\rtimes} \lambda^{\prime}=\zeta .
$$

From 233 and 25$]$, it follows that

$$
\psi\left(t^{*} d f\right)-\zeta=d \lambda^{\prime}-\partial^{\rtimes} \lambda^{\prime}
$$

Therefore we have $\left[\psi\left(t^{*} d f\right)-\zeta\right]=0$ in $H_{\mathrm{DR}}^{3}((\Gamma \rtimes G)$.).

Let $Q \in\left(\mathfrak{g}^{*} \otimes \Omega^{1}(X)\right)^{G}$ be defined by $\left.Q(\xi)=\widehat{\xi}\right\lrcorner B, \forall \xi \in \mathfrak{g}$.

Lemma 5.10. $\partial^{\rtimes} B-\operatorname{pr}^{*} \omega=\left(t^{\rtimes}\right)^{*} B-(t \circ p r)^{*} B=-\psi\left(t^{*} Q\right)$ 
Proof. The source and target maps of the groupoid $\Gamma \rtimes G \rightrightarrows X$ are given, respectively, by $s^{\rtimes}(\gamma, g)=s(\gamma)$ and $t^{\rtimes}(\gamma, g)=t(\gamma) \star g$. Using (19), we obtain

$$
\begin{aligned}
\partial^{\rtimes} B-\operatorname{pr}^{*} \omega & =\left(t^{\rtimes}\right)^{*} B-\left(s^{\rtimes}\right)^{*} B-\operatorname{pr}^{*}(\partial B) \\
& =\left(t^{\rtimes}\right)^{*} B-\left(s^{\rtimes}\right)^{*} B-\operatorname{pr}^{*}\left(t^{*} B-s^{*} B\right) \\
& =\left(t^{\rtimes}\right)^{*} B-(t \circ \operatorname{pr})^{*} B .
\end{aligned}
$$

This proves the first equality.

For the second equality, note that, by 150 and Lemma 5.4, we have

$$
\psi\left(t^{*} Q\right)=(t \times 1)^{*} \psi(Q)=(t \times 1)^{*}\left(s_{0}^{*} B-t_{0}^{*} B\right) .
$$

Here $s_{0}$ and $t_{0}$ are the source and target maps of the transformation groupoid $X \rtimes G \rightrightarrows$ $X$. It is clear that $t_{0} \circ(t \times 1)=t^{\rtimes}$ and $s_{0} \circ(t \times 1)=t \circ \mathrm{pr}$. Thus the second equality follows.

Proof of Theorem 5.6. Using 21, we get

$$
\mu\left(\eta_{G}\right)=\widehat{\pi}^{*} \psi(\alpha+\eta)=\pi^{*} \alpha+\widehat{\pi}^{*} \psi(\eta)=d B+\widehat{\pi}^{*} \psi(\eta) .
$$

And, since $\pi \circ t: \Gamma \rightarrow M$ is a $G$-equivariant map, we get

$$
\begin{array}{rlrl}
\widehat{\pi}^{*} \psi(\eta) & =((\pi \circ t) \times \mathrm{id})^{*} \psi(\eta) & \\
& =\psi\left((\pi \circ t)^{*} \eta\right) & & \text { by } 15 \\
& =\psi\left(t^{*} \pi^{*} \eta\right) & & \\
& =\psi\left(t^{*}(d f-Q)\right) & & \text { by } 22 \\
& =\psi\left(t^{*} d f\right)-\psi\left(t^{*} Q\right) . & &
\end{array}
$$

Therefore,

$$
\begin{aligned}
\mu\left(\eta_{G}\right) & =d B+\widehat{\pi}^{*} \psi(\eta) & & \text { by } 26 \\
& =d B+\psi\left(t^{*} d f\right)-\psi\left(t^{*} Q\right) & & \text { by } 27 \\
& =d B+\psi\left(t^{*} d f\right)+\partial^{\rtimes} B-\operatorname{pr}^{*} \omega & & \text { by Lemma } 5.10 .
\end{aligned}
$$

Hence, by Lemma 5.9 .

$$
\mu\left[\eta_{G}\right]=\left[d B+\partial^{\rtimes} B\right]+\left[\psi\left(t^{*} d f\right)-\operatorname{pr}^{*} \omega\right]=\left[\zeta-\operatorname{pr}^{*} \omega\right] .
$$

\section{Acknowledgements}

The author is indebted to Jean-Louis Tu and Ping Xu for providing access to their unpublished manuscript [16. This research work was supported by the European Union through the FP6 Marie Curie R.T.N. ENIGMA (Contract number MRTNCT-2004-5652). 


\section{References}

[1] K. Behrend and P. Xu, Differentiable Stacks and Gerbes (2006).

[2] J.-L. Brylinski, Loop spaces, characteristic classes and geometric quantization, Vol. 107 of Progress in Mathematics, Birkhäuser Boston Inc., Boston, MA (1993), ISBN 0-8176-3644-7.

[3] H. Bursztyn, M. Crainic, A. Weinstein, and C. Zhu, Integration of twisted Dirac brackets, Duke Math. J. 123 (2004), no. 3, 549-607.

[4] D. Chatterjee, On the construction of Abelian gerbes.

[5] M. Crainic, Differentiable and algebroid cohomology, van Est isomorphisms, and characteristic classes, Comment. Math. Helv. 78 (2003), no. 4, 681-721.

[6] J. L. Dupont, Curvature and characteristic classes, Springer-Verlag, Berlin (1978), ISBN 3-54008663-3. Lecture Notes in Mathematics, Vol. 640.

[7] K. Gawędzki and N. Reis, WZW branes and gerbes, Rev. Math. Phys. 14 (2002), no. 12, 12811334.

[8] J. Giraud, Cohomologie non abélienne, Springer-Verlag, Berlin (1971). Die Grundlehren der mathematischen Wissenschaften, Band 179.

[9] N. Hitchin, Lectures on special Lagrangian submanifolds, in Winter School on Mirror Symmetry, Vector Bundles and Lagrangian Submanifolds (Cambridge, MA, 1999), Vol. 23 of AMS/IP Stud. Adv. Math., 151-182, Amer. Math. Soc., Providence, RI (2001).

[10] L. C. Jeffrey, Group cohomology construction of the cohomology of moduli spaces of flat connections on 2-manifolds, Duke Math. J. 77 (1995), no. 2, 407-429.

[11] V. Mathai and D. Stevenson, Chern character in twisted $K$-theory: equivariant and holomorphic cases, Comm. Math. Phys. 236 (2003), no. 1, 161-186.

[12] E. Meinrenken, The basic gerbe over a compact simple Lie group, Enseign. Math. (2) 49 (2003), no. 3-4, 307-333.

[13] M. K. Murray, Bundle gerbes, J. London Math. Soc. (2) 54 (1996), no. 2, 403-416.

[14] M. K. Murray and D. Stevenson, Bundle gerbes: stable isomorphism and local theory, J. London Math. Soc. (2) 62 (2000), no. 3, 925-937.

[15] G. Segal, Classifying spaces and spectral sequences, Inst. Hautes Études Sci. Publ. Math. (1968), no. 34, 105-112.

[16] J.-L. Tu and P. Xu, Chern-Connes character and twisted equivariant cohomology, In preparation.

[17] J.-L. Tu, P. Xu, and C. Laurent-Gengoux, Twisted K-theory of differentiable stacks, Ann. Sci. École Norm. Sup. (4) 37 (2004), no. 6, 841-910.

Departement Mathematik, E.T.H. Zürich, RÄmistrasse 101, 8092 Zürich, Switzerland E-mail address: stienon@math.ethz.ch 